\title{
Causes, clinical and imaging characteristics of moyamoya disease in children.
}

\author{
Cai-Xia Wang ${ }^{1}$, Qing-Yi Wang ${ }^{2}$, Yi-Ming Zhao ${ }^{1}$, Mei Zhou ${ }^{1}$, Xiu-Xia Chen ${ }^{1}$, Rui-Yun Zhang ${ }^{1}$ \\ ${ }^{1}$ Department of Pediatrics, Qingdao Municipal Hospital, Qingdao, PR China \\ ${ }^{2}$ Department of Pain, Qingdao Municipal Hospital, Qingdao, PR China
}

\begin{abstract}
Introduction: Moyamoya disease is a progressive, chronic occlusive vascular disease of the circle of Willis arteries leading to the development of collateral circulation to compensate the occlusion.

Purpose: This study aims to investigate the potential causes and clinical and imaging characteristics of Moyamoya Disease (MMD) in children.

Methods: A retrospective analysis was performed on the clinical data of 14 MMD children, in which the potential causes were analysed, and the clinical and imaging characteristics were observed.

Results: The average patient age was $6.15 \mathrm{y}$ (range 21 months-13 y), and the female/male ratio was 1.3:1.0. There were 332 cases of ischemia and 196 hemorrhages. Eight cases had inducing factors including five cases of upper respiratory tract infection. Twelve cases exhibited limb weakness or hemiplegia. Among these patients, eight cases developed these manifestations as initial symptoms. Furthermore, seven cases presented language barriers, two cases had twitch, and two cases had headaches with unclear vision. All 14 patients underwent cranial Magnetic Resonance Imaging (MRI) and the results revealed that twelve cases had cerebral infarction, and two cases had cerebral hemorrhage. Five of 14 patients experienced stenosis or occlusion at the siphon part of the bilateral internal carotid arteries, seven patients had bilateral anterior cerebral artery stenosis or occlusion, 8 were bilateral middle cerebral artery stenosis or occlusion, and 2 were bilateral posterior cerebral artery stenosis or occlusion. All patients smoke-like vessels in the basal ganglia region.

Conclusion: High-risk age of child MMD ranged between 4-10 y old, which mostly manifested as transient cerebral ischemic insults; and inducing factors were mostly upper respiratory tract infections.
\end{abstract}

Keywords: Moyamoya disease, Children, Motivations, Clinical characteristic, Magnetic resonance angiography. Accepted on December 18, 2017

\section{Introduction}

Moyamoya syndrome is a disease in which certain arteries in the brain are constricted. Blood flow is blocked by the constriction, and also by blood clots (thrombosis) [1]. The moyamoya syndrome is a cerebrovascular condition that predisposes affected patients to stroke in association with progressive stenosis of the intracranial internal carotid arteries and their proximal branches. First described in 1957 as "hypoplasia of the bilateral internal carotid arteries," [2]. The characteristic appearance of the associated network of abnormally dilated collateral vessels on angiography was later likened to "something hazy, like a puff of cigarette smoke," [3], which, in Japanese, is moyamoya.

Moyamoya Disease (MMD), is a chronic, occlusive cerebrovascular disease involving bilateral stenosis or occlusion of the terminal portion of the Internal Carotid Arteries (ICAs) and/or the proximal portions of the anterior cerebral arteries and Middle Cerebral Arteries (MCAs). Moyamoya disease is also characterized by irregular perforating vascular networks, called moyamoya vessels, near the occluded or stenotic regions corresponding to the lenticulostriate and thalamoperforate arteries. This makes the blood leak out of the arteries, causing pressure to the brain and subsequent headaches. The Ministry of Health and Welfare of Japan has defined 4 types of moyamoya disease (MMD): ischemic, hemorrhagic, epileptic, and "other." The ischemic type has been shown to predominate in childhood, while the hemorrhagic type is more often observed in the adult population.

Moyamoya Disease (MMD), by the appearance of smoke on relevant angiographs resultant from the tangle of tiny vessels in response to stenosis. This makes the blood leak out of the arteries, causing pressure to the brain and subsequent headaches. Though the research of moyamoya disease has made great strides in the past 60 years, the etiology and pathogenesis are largely unknown. The pathogenesis of moyamoya disease is unknown, although the gene Ring Finger Protein 213 (RNF213) has been implicated [4]. In Japan, the incidence of Moyamoya Disease (MMD) is $1 / 1000,000$ [5]. There are two onset peaks in age: under $10 \mathrm{y}$ old and within 
$30-40$ y old; and the ratio of male to female patients is approximately $1: 1.7$.

The diagnosis of moyamoya is suggested by CT, MRI, or angiogram results. Contrast-enhanced T1-weighted images are better than FLAIR images for depicting the leptomeningeal ivy sign in moyamoya disease. MRI and MRA should be performed for the diagnosis and follow-up of moyamoya disease. Diffusion-weighted imaging can also be used for following the clinical course of children with moyamoya disease, in whom new focal deficits are highly suspicious of new infarcts.

The natural history of this disorder is not well known. The long term outlook for patients with treated moyamoya seems to be good.

Recent investigations have established that both moyamoya disease and Arteriovenous Fistulas (AVFs) of the lining of the brain, the dura, are associated with dural angiogenesis. These factors may represent a mechanism for ischemia contributing to the formation of dural AVFs [6].

With the development of Magnetic Resonance Imaging (MRI) diagnostic techniques, reports in China on MMD have gradually increased in recent years. In our hospital, 14 hospitalized children were confirmed with this disease from July 2003 to June 2008. Details are reported as follows.

\section{Materials and Methods}

\section{Case data}

A total of 14 hospitalized children diagnosed with MMD by clinical information combined with imaging tests from July 2000 to June 2008 were included into this study. The age of these children ranged between 21 months and $13 \mathrm{y}$ old, with an average age of $6.15 \mathrm{y}$ old. Among them, the age of 11 children ranged between 21 months and 10 y old (78.5\%). Furthermore, among these 14 children, eight children were female and six children were male, with a female-to-male ratio of 1.3:1. The course of the disease ranged from $1.5 \mathrm{~h}$ to four years. Seven children went to see a doctor after the first onset, five children went to see a doctor after the second onset, and two children went to see a doctor after the third onset. Five children were diagnosed with viral encephalitis at the first diagnosis, one child was diagnosed with mycoplasma encephalitis, and three children were diagnosed with acute hemiplegia syndrome.

\section{Methods}

Clinical data of 14 children were retrospectively summarized. Adjuvant examinations: Six children underwent cranial Computed Tomography (CT) examinations, and all 14 patients underwent cranial MRI and Magnetic Resonance Angiography (MRA). Furthermore, three children underwent Digital Subtraction Angiography (DSA), Eight Children Underwent Electroencephalography (EEG) examinations, and six children received cerebrospinal fluid tests. In addition, all 14 children were tested for tuberculin, blood sedimentation, C-reactive protein and blood coagulation function, Antistreptolysin $\mathrm{O}$ (ASO), complement $\mathrm{C} 3$, antinuclear antibodies and antineutrophils; while eight children received serum Epstein-Barr Virus (EBV), herpes simplex virus, and rubella virus antibody tests. Moreover, six children received mycoplasma antibody tests.

\section{Diagnostic criteria}

The radiological diagnosis criteria proposed in 1997 by the Moyamoya Disease Research Committee of the Japanese Ministry of Health and Welfare was used as a reference [7]: (1) stenosis or occlusion exist in the end of the internal carotid artery, or in the initial segments of the middle and anterior cerebral arteries; (2) the arteries of the skull base are filled, and the surroundings of the blockings exhibit abnormal blood vessel nets; (3) bilateral involvement. When the above three conditions are met and systemic disease is excluded, MMD diagnosis can be established. In MMD children, smoke-like vessels sometimes primarily appear on one side, and the contralateral artery develops lesions with the increase of age. Therefore, unilateral lesions are also considered in children for MMD.

\section{Results}

\section{Clinical characteristics}

We identified 14 patients who met the criteria for study inclusion. The age of these children ranged between 21 months and $13 \mathrm{y}$ old, with an average age of $6.15 \mathrm{y}$ old. Among them, the age of 11 children ranged between 21 months and $10 \mathrm{y}$ old (78.5\%). Furthermore, among these 14 children, eight children were female and six children were male, with a female-to-male ratio of 1.3:1. The course of the disease ranged from $1.5 \mathrm{~h}$ to four years. Inducing factors: Eight children had inducing factors including five cases of upper respiratory tract infections (four cases had fever and one case had cough). Among these children, three children with fever developed limb weakness or paralysis within three days, and one child with fever developed limb weakness four days after the fever. Furthermore, two children fell and developed paralysis on the same day, while one case was induced by intense crying.

Initial symptoms: Eight children exhibited limb weakness or hemiplegia, two children exhibited twitch, and two children developed unclear vision. Furthermore, one child had conscious disturbance after headaches and vomiting, and one child had hearing loss.

Clinical manifestations: Twelve children exhibited limb weakness or hemiplegia, among which 10 cases had repetitive limb weakness or hemiplegia, three cases had alternate episodes, and seven cases exhibited unilateral involvement. Furthermore, five cases developed persistent hemiplegia from onset, while five cases first exhibited repetitive episodes and gradually progressed to persistent hemiplegia. In addition, seven cases exhibited distortion of commissure, facial paralysis and unclear articulation, two cases exhibited twitch, two cases 
involuntary movement of extremities with numbness, and two cases had headaches with unclear vision. Among these 14 patients, five patients had a history of infection before onset, and one patient had head trauma five years before the onset of MMD. Furthermore, one case was combined with cutis marmorata telangiectatica congenita, one case had hyperthyroidism, and one case had chicken pox viral encephalitis six month before onset. Moreover, two cases had a family history of cerebrovascular disease, and the mother of one child with cutis marmorata telangiectatica congenita also had the same disease.

\section{Adjuvant examination}

Neural imageologic tests: Six children received cranial CT examinations, and all children were found to have cerebral ischemic changes. All 14 children received cranial MRI and MRA examinations. MRI manifestations: Twelve cases had cerebral infarction, and two cases had cerebral hemorrhage. Cerebral infarction manifested as infarcts or softened lesions. Eight cases had encephalatrophy and seven cases had ventriculomegaly. Lesions were characterized by long T1 and T2 signals. Nine cases exhibited bilateral MMD in MRA images, and five cases had unilateral MMD. Furthermore, five cases had stenosis or occlusion in the siphon part of bilateral internal carotid arteries, seven cases had stenosis or occlusion in the bilateral anterior cerebral arteries, eight cases had stenosis or occlusion in the bilateral middle cerebral arteries, and two cases had stenosis or occlusion in the bilateral anterior cerebral arteries. In addition, five cases had an unclear display of the anterior communicating artery, and four cases had an unclear display of the posterior communicating artery. Moreover, 14 cases had smoke-like vessels in the basal ganglia region. Three children underwent DSA examinations, and these results were consistent with those of MRA. Both MRI and MRA cranial examination results of one child are shown in Figure 1.

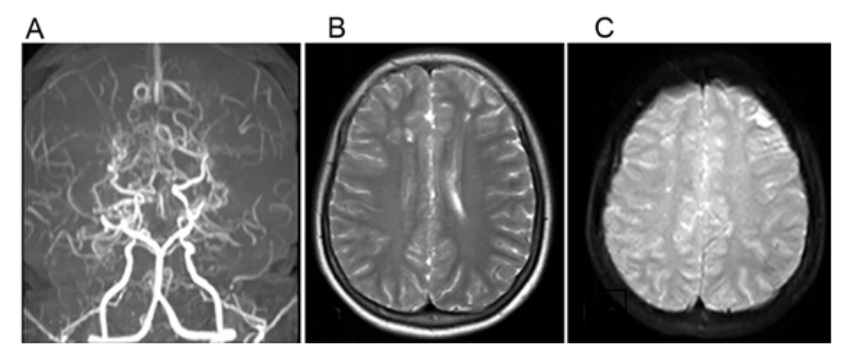

Figure 1. Examining results of one case in MRI, MRA. A: examination result of MRA suggested that inner segment of bilateral internal carotid artery became thin, A1 segment of bilateral anterior cerebral artery and proximal segment of cerebral middle artery blocked and massive compensatory enlargement veins like smoke were also observed. $B$ and $C$ : examining results of MRI T2W1 and DW1 showed multiple long T2 and high signal in the bilateral ventricle and bilateral parietal and under the cortex of right occipital lobe.

Other adjuvant examinations: Eight children received electroencephalography examinations and two cases with twitch exhibited focus-derived abnormal discharges. In the remaining six patients, five patients exhibited abnormal slowwave band enhancement. Furthermore, six patients received cerebrospinal fluid examinations, in which two children had a slightly increased cell count $\left(0.016 \times 10^{9}\right.$ and $0.012 \times 10^{9}$, respectively). In 14 cases, white blood cell count increased in five cases; which were 18.7, 16.2, 12.1, 11.8 and $10.3 \times 10^{9}$, respectively (normal cell count is 3.6-9.2 $\times 10^{9}$ ). Blood sedimentation was conducted in 14 cases, and was found to increase to $25 \mathrm{~mm} / \mathrm{h}$ in one case (normal: $<15 \mathrm{~mm} / \mathrm{h}$ for male and $<20 \mathrm{~mm} / \mathrm{h}$ female). Six cases underwent mycoplasma pneumoniae antibody detection, in which three cases had abnormal results (results for the two cases were both 1:40, and the result for the other case was 1:80). Eight cases received rubella virus antibody detection, in which three cases were found as positive (one case was found positive for cerebrospinal fluid mycoplasma pneumoniae antibody). In addition, EBV and herpes simplex virus antibody tests revealed negative results in three cases. In all 14 children, tuberculin, blood sedimentation, C-reactive protein and blood coagulation function, ASO, complement $\mathrm{C} 3$, antinuclear antibodies and anti-neutrophils tests revealed no abnormal results.

\section{Treatment and prognosis}

Among the 14 children with proven diagnosis, one child died of serious cranial high pressure and respiratory failure caused by cerebral infarction; and the remaining children received internal medicine conservative treatment. Nutrition for brain cells, intracraninal pressure lowering by mannitol and furosemide or a small dose of hormone, as well as antiinflammation, expansion of blood vessels and calcium channel antagonist treatments, were performed. In addition, rehabilitation, functional exercise, psychological therapy and special education were provided according to the conditions of the children. Among these children, three cases received vasodilatation within 24 hours after onset of the disease.

\section{Follow-ups}

Among the 14 patients, the one who was 12 y old boy, had stenosis of bilateral internal carotid arteries, experienced 3times extensive cerebral infarction, died 6 months later after onset, and the others survived. The 13 survived patients continuously take small dose aspirin and dipyridamole orally. Among the rest 13 patients, 4 patients suffered hemiplegia, 2 walked unsteadily and the others suffered no hemiplegia. Among 4 hemiplegic patients, one is 21 -months girl, one is $4 \mathrm{y}$ old boy, one is $6 \mathrm{y}$ old boy and one is $11 \mathrm{y}$ old boy; 3 patients with bilateral internal carotid artery stenosis and one patient with bilateral middle cerebral artery stenosis; 2 patients with the left lower limb paralysis and 2 patients with the right lower limb paralysis; the 6 y old boy with light dysgnosia by Gesell and the other three normal. Among the 2 patients who walked unsteadily, one is $5 \mathrm{y}$ old boy and the other one is $8 \mathrm{y}$ old boy, and they have normal intelligence and can walk by stick and wheelchair. Among 7 patients who have no hemiplegia, only 
one had mild mental retardation and 4 patients with bilateral internal carotid artery stenosis were given the medical support.

\section{Discussion}

The prevalence of the disease among the Nanjing in China was $3.92 / 100,000$, as reported by Miao et al. in 2010, showing a rising trend [8]. Moyamoya disease cases typically present acutely with various cerebrovascular events including intracranial hemorrhage, TIAs, brain infarction, and sometimes epileptic seizures. The Ministry of Health and Welfare of Japan has defined 4 types of MMD with the following presentation percentages: ischemic $63.4 \%$, hemorrhagic $21.6 \%$, epileptic $7.6 \%$, and "other" $7.5 \%$ [9]. As reported before, the ischemic type of MMD predominates in childhood, making up $69 \%$ of cases in patients under 10 years old, which is consistent with our results [10]. However, the epidemiological features of MMD determined by this study differed from those in previous reports. In the previous literature, women have a higher incidence than men, with the female/male predominance ranging from $1.8: 1$ to $4.25: 1$. In the present study, the ratio of girls to boys was $1.3: 1$, which is much lower than the ratio reported compared with the other Asian and Western countries. This might reveal that MMD has been associated with the agespecific ratio patterns [11-14].

In our study, all 14 children received cranial MRI and MRA examinations, in which 12 cases were found to have cerebral ischemia changes, and two cases were found to have cerebral hemorrhage. MRA revealed stenosis or occlusion in internal carotid artery, as well as in the anterior, middle and posterior cerebral arteries; and abnormalities in the anterior and middle cerebral arteries were the most common. Furthermore, smokelike vessels mostly occurred in the basal ganglia region. Three children received DSA examination, and the results were consistent with those obtained by MRA. In this study, MRI combined with MRA examination can not only display vascular lesions, but also show cerebral infarction, cerebromalacia, cerebral hemorrhage and cerebral atrophy; which is secondary to vascular lesions. Furthermore, it is convenient, noninvasive, does not need a contrast agent, and is conducive to long-term follow-ups. Some literatures have revealed that MRA examination has certain limitations such as low spatial resolution, exaggeration of the extent of the vascular occlusion, and poor display of intracranial collateral circulation. Therefore, in MMD patients who require surgery, DSA still needs to be performed in order clearly examine these specific sites.

The cause of MMD remains unclear. At present, the following views have been reported. (1) Genetic factors. The epidemiological characteristics of MMD prompt the onset of the disease, as a kind of polygenic hereditary disease. Studies have found that [15-17] chromosomes 3p24, 2-p26, 6q, 17q25 and $8 \mathrm{q} 23$ were related to familial MMD. Characteristics of genetic moyamoya syndromes are presented, with a focus on recently reported mutations in $B R C C 3 / M T C P 1$ and $G U C Y 1 A 3$ genes and Liu et al. provided evidence suggesting, for the first time, the involvement of RNF213 in genetic susceptibility to moyamoya disease. However, the mechanisms leading from RNF213 mutations to MMD clinical features are still unknown [18]. (2) Inflammation and immune responses. A variety of acquired factors such as EBV infection, cerebral trauma, tuberculous meningitis, and multiple Takayasu arteritis and radiation injury can cause chronic inflammation of the central nervous system; and result in MMD [19]. Besides, the study [20] also shows thyroid storm may trigger cerebral ischemia in Moyamoya disease and lead to rapid progression of cerebrovascular occlusive disease. Greco et al. [21] reported cases of mycoplasma pneumoniae infection followed by MMD. In the present study, six children underwent mycoplasma pneumoniae antibody detection, and three cases revealed positive results. Among these three cases, one case was positive for cerebrospinal fluid mycoplasma pneumoniae antibody; suggesting that mycoplasma pneumoniae infection might be correlated with MMD. Furthermore, in this study, one child developed chicken pox viral encephalitis six months before the occurrence of MMD. This case should be given attention, as the study of Makoto et al. [22] also reported one case of MMD caused by chicken pox virus infection. Secondary MMD is also known as moyamoya syndrome. Some literatures have reported that MMD co-existed with systemic lupus erythematosus, AIDS, Behcet's disease, multiple Takayasu arteritis, hyperthyroidism and cutis marmorata telangiectatica congenita. In this study, one child and the mother both developed cutis marmorata telangiectatica congenita. In MMD, cell proliferation and vascular dysfunction are associated with $\mathrm{T}$ cell immune dysfunction. In the above cases, immune complex injury following infections or autoimmune injury has been considered. That is, the intravascular elastic layer is curved and broken, and the medial smooth muscle proliferates towards the intima at the break site. These would result in luminal stenosis or occlusion, and chronic ischemia in brain tissues at the artery distal end; and subsequently, compensatory hyperplasia of small blood vessels and capillaries. Hemorrhagic MMD has two major imaging features: the dilatation and abnormal branching of anterior choroidal artery or posterior communicating artery, and multiple microbleeds, which may predict subsequent hemorrhage [23].

It has been reported in the previous literature that this disease mostly occurred when crying, eating hot food, blowing balloons and other excessive breathing activities; and symptoms continuously re-appear. Moyamoya Disease (MMD) is a chronic, occlusive cerebrovascular disease characterized by progressive stenosis at the terminal portion of the internal carotid artery and an abnormal vascular network at the base of the brain [24]. Since both sides of the brain are affected, the clinical syndromes initially manifest as vascular Parkinsonism [25], meanwhile hemiplegia, intelligence decline, seizures and headaches are observed usually. Therefore, to improve the quality of life of MMD patients not only the protection from the neurological insult but also the maintenance or improvement of cognitive function is inevitable [26]; which alternatively appear in the left and right side. In this study, the average age of onset was $6.15 \mathrm{y}$ old, which belong to the 
school-age onset. Eight children were female and six children were male. Before the disease, crying and fatigue were the incentives. It is noteworthy to mention that, in this study, five children developed upper respiratory tract infections before the onset of MMD; reminding us to determine whether infection is the cause of the disease and which one is worth to be further discussed.

MMD can affect both children and adults, but MMD in pediatric patients exhibits distinct clinical features, and the treatment prognoses are different from adult patients. Children are the group at highest risk for MMD. In children, the disease mainly manifests as ischemia, while bleeding is the primary symptom in adults [27].The onset age of the disease is mostly under $10 \mathrm{y}$ old, and the average age is approximately $4.5 \mathrm{y}$ old. Japanese girls are more prone to this disease. In adults, cerebral hemorrhage is the main symptom; while cerebral ischemia is the initial symptom, which occurs in $95 \%$ of children. In this study, 21 month-old to $10 \mathrm{y}$ old children accounted for $78.5 \%$. MRI revealed that 12 cases had cerebral ischemia, and two cases had cerebral hemorrhage; which is basically consistent with that reported in Southeast Asia [28]. In this study, 12 of 14 patients had the onset with limb weakness or hemiplegia, which accounted for $85.7 \%$. Among these 12 patients, 10 patients had repeated episodes, which accounted for $71.4 \%$. Five cases exhibited persistent hemiplegia at the beginning of the disease, prompting that hemiplegia is the most common clinical manifestation in children with MMD. Other manifestations are unclear vision, sensory disturbance, headaches, epileptic seizure, and involuntary movement. These prompt that limb weakness or hemiplegia, sensory disturbance, and language barrier are the main manifestations of MMD in children. Especially for patients with repetitive limb weakness or hemiplegia, timely cranial imageologic tests should be given.

DSA is the gold standard for the diagnosis of MMD, but it is an invasive test. At present, DSA has not been accepted by most families of these diseased children. The application of MRI and MRA provides a non-invasive diagnostic method for the vascular pathological examination of MMD. All 14 children received cranial MRI and MRA examinations, in which 12 cases were found to have cerebral ischemia changes, and two cases were found to have cerebral hemorrhage. MRA revealed stenosis or occlusion in internal carotid artery, as well as in the anterior, middle and posterior cerebral arteries; and abnormalities in the anterior and middle cerebral arteries were the most common. Furthermore, smoke-like vessels mostly occurred in the basal ganglia region. Three children received DSA examination, and the results were consistent with those obtained by MRA. In this study, MRI combined with MRA examination can not only display vascular lesions, but also show cerebral infarction, cerebromalacia, cerebral hemorrhage and cerebral atrophy; which is secondary to vascular lesions. Furthermore, it is convenient, noninvasive, does not need a contrast agent, and is conducive to long-term follow-ups. Some literatures have revealed that MRA examination has certain limitations such as low spatial resolution, exaggeration of the extent of the vascular occlusion, and poor display of intracranial collateral circulation. Therefore, in MMD patients who require surgery, DSA still needs to be performed in order clearly examine these specific sites.

In this study, we performed conservative treatment for patients with ischemia rather than surgical treatment. Conservative treatment is mainly symptomatic treatment. For patients with ischemia, capacity expansion, blood vessel expansion and intracraninal pressure lowering treatment should be given as soon as possible (within a week, preferably within $24 \mathrm{~h}$ ). If there are any infection factors, anti-infection treatment should be actively given. In recent years, surgical treatment has presented a good effect. Especially, the effectiveness of surgical revascularization for preventing ischemic stroke had been generally accepted by many studies [29]. It also has been reported that patients with hemorrhagic MMD had a much higher rate of rebleeding and poorer prognosis than those with the ischemic type. Surgical revascularization procedures can improve cerebral perfusion and have a positive impact in preventing rebleeding in patients with hemorrhagic MMD [30]. However, in our study, the treatment conservatively for the patient with the ischemic types has been proved effective.

In conclusion, high-risk age of child MMD ranged between 4-10 y old, which mostly manifested as transient cerebral ischemic insults; and inducing factors were mostly upper respiratory tract infections. We believe that genetic research for the susceptibility gene such as RNF213 would be more supportive to lead to the definitive diagnosis of moyamoya disease in the future if the genetic analysis of such susceptibility gene becomes generally available in each clinic.

\section{References}

1. Scott RM, Smith ER. Moyamoya disease and moyamoya syndrome. N Engl J Med 2009; 360: 1226-1237.

2. Takeuchi K, Shimizu K. Hypoplasia of the bilateral internal carotid arteries. Brain Nerve 1957; 9: 37-43.

3. Suzuki J, Takaku A. Cerebrovascular moyamoya disease. Disease showing abnormal net-like vessels in base of brain. Arch Neurol 1969; 20: 288-299.

4. Ma J, Liu Y, Ma L, Huang S, Li H, You C. RNF213 polymorphism and Moyamoya disease: A systematic review and meta-analysis. Neurol India 2013; 61: 35-39.

5. Fukui M. Current state of study on moyamoya disease in Japan. Surg Neurol 1997; 47: 138-143.

6. Killory BD, Gonzalez LF, Wait SD, Ponce FA, Albuquerque FC, Spetzler RF. Simultaneous unilateral moyamoya disease and ipsilateral dural arteriovenous fistula: case report. Neurosurgery 2008; 62: 1375-1376.

7. Fukui M. Guideline for the diagnosis and treatment of spontaneous occlusion of the Circle of Willis (Moyamoya Disease) of the Ministry of Health and Welfare, Japan. Clin Neuro Surg 1997; 99: 238-240.

8. Miao W, Zhao PL, Zhang YS, Liu HY, Chang Y, Ma J, Huang QJ, Lou ZX. Epidemiological and clinical features of Moyamoya disease in Nanjing, China. Clin Neurol Neurosurg 2010; 112: 199-203. 
9. Fukui M. Current state of study on moyamoya disease in Japan. Surg Neurol 1997; 47: 138-143.

10. Cho HJ, Jung YH, Kim YD, Nam HS, Kim DS, Heo JH. The different infarct patterns between adulthood-onset and childhood-onset moyamoya disease. J Neurol Neurosurg Psychiatry 2011; 82: 38-40.

11. Baba T, Houkin K, Kuroda S. Novel epidemiological features of moyamoya disease. J Neurol Neurosurg Psychiatry 2008; 79: 900-904.

12. Chiu D, Shedden P, Bratina P, Grotta JC. Clinical features of moyamoya disease in the United States. Stroke 1998; 29: 1347-1351.

13. Hallemeier CL, Rich KM, Grubb RL, Chicoine MR, Moran CJ, Cross DT, Zipfel GJ, Dacey RG, Derdeyn CP. Clinical features and outcome in North American adults with moyamoya phenomenon.Stroke 2006; 37: 1490-1496.

14. Kraemer M, Heienbrok W, Berlit P. Moyamoya disease in Europeans. Stroke 2008; 39: 3193-3200.

15. Inoue TK, Ikezaki K, Sasazuki T, Matsushima T, Fukui M. Linkage analysis of moyamoya disease on chromosome $6 . \mathrm{J}$ Child Neurol 2000; 15: 179-182.

16. Guey S, Tournier-Lasserve E, Hervé D, Kossorotoff $M$. Moyamoya disease and syndromes: from genetics to clinical management. Appl Clin Genet 2015; 8: 49-68.

17. Liu W, Morito D, Takashima S, Mineharu Y, Kobayashi H, Hitomi T, Hashikata H, Matsuura N, Yamazaki S, Toyoda A, Kikuta K, Takagi Y, Harada KH, Fujiyama A, Herzig R, Krischek B, Zou L, Kim JE, Kitakaze M, Miyamoto S, Nagata K, Hashimoto N, Koizumi A. Identification of RNF213 as a susceptibility gene for moyamoya disease and its possible role in vascular development. PLoS One 2011; 6: 22542 .

18. Bersano A, Guey S, Bedini G, Nava S, Herve D, Vajkoczy P, Tatlisumak T, Sareela M, van der Zwan A, Klijn CJ, Braun KP, Kronenburg A, Acerbi F, Brown MM, Calviere L, Cordonnier C, Henon H, Thines L, Khan N, Czabanka M, Kraemer M, Simister R, Prontera P, Tournier-Lasserve E, Parati E, EuropeanMoyamoya Disease Initiative. Research progresses in understanding the pathophysiology of moyamoya disease. Cerebrovasc Dis 2016; 41: 105-118.

19. Kirkham FJ, Hogan AM. Risk factors for arterial ischemic stroke in childhood. CNS Spectr 2004; 9: 451-464.

20. Noh BH, Cho SW, Ahn SY. Simultaneous occurrence of diabetic ketoacidosis, thyroid storm, and multiple cerebral infarctions due to Moyamoya disease. J Pediatr Endocrinol Metabol 2016; 29: 221-225.

21. Greco F, Castellano Chiodo D, Sorge A, Perrini S, Sorge G. Multiple arterial ischemic stroke in a child with moyamoya disease and Mycoplasma pneumoniae infection. Minerva Pediatr 2006: 58: 63-68.

22. Ueno M, Oka A, Koeda T, Okamoto R, Takeshita K. Unilateral occlusion of the middle cerebral artery after varicella-zoster virus infection. Brain Develop 2002; 24: 106-108.

23. Wan M, Duan L. Recent progress in hemorrhagic moyamoya disease. Br J Neurosurg 2015; 29: 189-191.

24. Kim JS. Moyamoya disease: epidemiology, clinical features, and diagnosis. J Stroke 2016; 18: 2-11.

25. Leea TH, Sungb SM, Chob HJ. Moyamoya disease initially manifesting as vascular parkinsonism. J Neurol Sci 2015; 357: 412-413.

26. Shim KW, Park EK, Kim JS, Kim DS. Cognitive outcome of pediatric moyamoya disease. J Korean Neurosurg Soc 2015; 57: 440-444.

27. Piao J, Wu W, Yang Z, Yu J. Research progress of moyamoya disease in children. Int J Med Sci 2015; 12: 566-575.

28. Morioka M, Hamada J, Todaka T, Yano S, Kai Y, Ushio Y. High-risk age for rebleeding in patients with hemorrhagic moyamoya disease: long-term follow-up study. Neurosurgery 2003; 52: 1049-1054.

29. Kim T, Oh CW, Bang JS, Kim JE, Cho WS. Moyamoya disease: treatment and outcomes. J Stroke 2016; 18: 21-30.

30. Liu XJ, Zhang D, Wang S, Zhao YL, Teo M, Wang R, Cao Y, Ye X, Kang S, Zhao JZ. Clinical features and long-term outcomes of moyamoya disease: a single-center experience with 528 cases in China. J Neurosurg 2015; 122: 392-399.

\section{*Correspondence to}

Rui-Yun Zhang

Department of Pediatrics

Qingdao Municipal Hospital

PR China 